\title{
Temperature-Profile/Lapse-Rate Feedback: A Misunderstood Feedback of the Climate System
}

\author{
Michael E. Schlesinger ${ }^{1}$, C. Bruce Entwistle ${ }^{2}$, Bin $\mathrm{Li}^{3}$ \\ ${ }^{1}$ Climate Research Group, Department of Atmospheric Sciences, University of Illinois at Urbana-Champaign, Urbana, USA \\ ${ }^{2}$ Aviation Weather Center, National Weather Service, Kansas City, USA \\ ${ }^{3}$ I. M. Systems Group, Inc. at EMC/NCEP/NOAA, College Park, USA \\ Email: schlesin@atmos.uiuc.edu
}

Received July 19, 2012; revised August 20, 2012; accepted August 31, 2012

\begin{abstract}
This study shows that the heretofore assumed condition for no temperature-profile (TP)/lapse-rate feedback, $\Delta T(z)=\Delta T_{s}$ for all altitudes $\mathrm{z}$, or $\Delta(\mathrm{d} T(z) / \mathrm{d} z)=0$, in fact yields a negative feedback. The correct condition for no TP feedback is $\Delta T(z) / T(z)=\Delta T_{s} / T_{s}$ for all $\mathrm{z}$, where $T_{s}$ is the surface temperature. This condition translates into a uniform increase (decrease) in lapse rate with altitude for an increase (decrease) in $T_{s}$. The temperature changes caused by a change in solar irradiance and/or planetary albedo satisfy the condition for no TP feedback. The temperature changes caused by a change in greenhouse gas concentration do not satisfy the condition for no TP feedback and, instead, yield a positive feedback.
\end{abstract}

Keywords: Climate Feedback; Feedback Analysis; Lapse-Rate Feedback

\section{Introduction}

Feedback due to changes in the vertical temperature profile has been called lapse-rate feedback [1]. It has been assumed that the condition for this feedback to be zero is that $\Delta T(z)=\Delta T_{s}$ for all altitudes $z$, where $T(z)$ is the temperature at $\mathrm{z}$ and $T_{s}$ is the surface temperature [2]. This condition is equivalent to $\Delta(\mathrm{d} T(z) / \mathrm{d} z)=0$, that is, no change in lapse rate, hence the name lapse-rate feedback when $\Delta(\mathrm{d} T(z) / \mathrm{d} z) \neq 0$. Here we first use a one-layer atmospheric model, and then a multilayer atmospheric model, to show what we found 20 years ago [3], namely, that the correct answer for zero feedback is $\Delta \log T(z)=\Delta \log T_{s}$ or, equivalently,

$$
\Delta T(z) / T(z)=\Delta T_{s} / T_{s}
$$

for all $z$. When this condition is not satisfied, there is feedback. In particular, if $\Delta T(z)=\Delta T_{s}$ for all altitudes $z$, the feedback is negative. Since there is feedback when the lapse rate does not change, it is recommended that the name lapse-rate feedback be supplanted by the appellation temperature-profile (TP) feedback.

\section{Feedback Analysis}

This section is based on the feedback analysis of Schlesinger [4-6]. The net downward radiation at the top of the atmosphere (TOA) per unit area, $N$, is given by

$$
N=\left(\frac{1-\alpha_{p}}{4}\right) S-\varepsilon_{p} \sigma T_{s}^{4}
$$

where $\mathrm{S}$ is the solar irradiance at TOA, $\alpha_{p}$ is the planetary albedo, $\sigma$ is the Stefan-Boltzmann constant, and $\varepsilon_{p}$ is the planetary emissivity. The change in $\mathrm{N}$ due to external radiative forcing $F$, say due to a change in $\mathrm{S}$ or the anthropogenic increase in the concentrations of greenhouse gases, can be written as

$$
\Delta N=F+\left[\frac{\partial N}{\partial T_{s}}+\sum_{j} \frac{\partial N}{\partial I_{j}} \frac{\mathrm{d} I_{j}}{\mathrm{~d} T_{s}}\right] \Delta T_{s}
$$

where the second term on the right-hand side is the change in $\mathrm{N}$ due to the change in $T_{s}$ alone, and the third term is the change in $\mathrm{N}$ due to the change in internal quantities $I_{j}$ - such as the temperature profile; water vapor amount; cloud amount, height and optical depththrough their dependence on $T_{s}$. From Equation (1) we can also write

$$
\Delta N=F-\left[4 \varepsilon_{p} \sigma T_{s}^{3}+\left(\frac{S}{4} \frac{\Delta \alpha_{p}}{\Delta T_{s}}+\frac{\Delta \varepsilon_{p}}{\Delta T_{s}} \sigma T_{s}^{4}\right)\right] \Delta T_{s}
$$

For the new equilibrium, $\Delta N=0$, hence by Equation (2) we have

$$
\Delta T_{s}=G_{f} F=\left(\frac{G_{o}}{1-f}\right) F
$$


where

$$
G_{f}=\frac{G_{o}}{1-f}
$$

is the gain of the climate system with feedback,

$$
f=G_{o} \sum_{j} \frac{\partial N}{\partial I_{j}} \frac{\mathrm{d} I_{j}}{\mathrm{~d} T_{s}}
$$

and $G_{o}$ is the gain of the climate system with zero feedback $(f=0)$. From Equation (3) with $\Delta N=0$ and

$$
\Delta \varepsilon_{p}=\Delta \alpha_{p}=0
$$

we obtain,

$$
G_{o}=-\left(\frac{\partial N}{\partial T_{s}}\right)^{-1}=\frac{1}{4 \varepsilon_{p} \sigma T_{s}^{3}}=\frac{T_{s}}{\left(1-\alpha_{p}\right) S}
$$

the latter from Equation (1) with $N=0$, that is, the equilibrium before the radiative forcing $\mathrm{F}$ is applied. For present-day conditions, prescribed in Table 1 and calculated in Table 2, $G_{o}=0.30 \mathrm{~K} / \mathrm{Wm}^{-2}$. Thus if the climate system had zero feedback, the temperature change due to a doubling of the $\mathrm{CO}_{2}$ concentration would be $\left(\Delta T_{2 x}\right)_{o}=G_{o} F_{2 x}=1.11^{\circ} \mathrm{C}$ for $F_{2 x}=3.71 \mathrm{Wm}^{-2}$ [7].

From Equation (6) and Equations (2) and (3) with $\Delta N=0$ it can be seen that

$$
f=-G_{o}\left(\frac{S}{4} \frac{\Delta \alpha_{p}}{\Delta T_{s}}+\frac{\Delta \varepsilon_{p}}{\Delta T_{s}} \sigma T_{s}^{4}\right)
$$

For the feedback we consider here, $\Delta \alpha_{p}=0$. Thus, if $\varepsilon_{p}$ increases (decreases) with increasing (decreasing) $T_{s}$, $\Delta \varepsilon_{p} / \Delta T_{s}>0$, the change in emissivity works to decrease (increase) $\Delta T_{s}$, hence as shown by Equation (8), $f<$ 0 . Conversely, if $\varepsilon_{p}$ decreases (increases) with increasing (decreasing) $T_{s}, \Delta \varepsilon_{p} / \Delta T_{s}<0$, the change in emissivity works to increase (decrease) $\Delta T_{s}$, hence as shown by Equation (8), $f>0$. Below we show that the heretofore assumed condition for zero feedback,

$$
\Delta T(z)=\Delta T_{s}
$$

for all altitudes $z$, actually yields a negative feedback, $f<0$.

Table 1. Prescribed quantities.

\begin{tabular}{cc}
\hline Quantity & Value \\
\hline Stefan-Boltzmann constant, $\sigma$ & $5.67 \times 10^{-8} \mathrm{Wm}^{-2} \cdot \mathrm{K}^{-4}$ \\
Solar irradiance, $S$ & $1367 \mathrm{Wm}^{-2}$ \\
Planetary albedo, $\alpha_{p}$ & 0.3 \\
Longwave absorptance, $a$ & 0.8 \\
Solar absorptance, $b$ & 0.1 \\
Change in $R^{\uparrow}$ due to a $\mathrm{CO}_{2}$ doubling & $-3.71 \mathrm{Wm}^{-2}[7]$ \\
\hline
\end{tabular}

\section{Condition for No Temperature-Profile Feedback}

Consider the one-layer atmosphere shown in Figure 1 with temperature, $T_{a}$, infrared (IR) absorptance, $a$, and solar absorptance $b$. Energy balance at TOA and the sur-

\begin{tabular}{|c|c|c|}
\hline Quantity & Equation & Value \\
\hline Planetary emissivity, $\varepsilon_{p}$ & (1) with $N=0$ & 0.63 \\
\hline Equivalent blackbody temperature, $T_{e}$ & (11) & $254.86 \mathrm{~K}$ \\
\hline Surface temperature, $T_{s}$ & (12) & $285.89 \mathrm{~K}$ \\
\hline Atmosphere temperature, $T_{a}$ & (13) & $245.01 \mathrm{~K}$ \\
\hline $\begin{array}{l}\text { Gain of the climate system without } \\
\text { feedback, } G_{o}\end{array}$ & (7) & 0.299 \\
\hline $\begin{array}{l}\text { Change in longwave absorptance due } \\
\text { to a } \mathrm{CO} 2 \text { doubling, } \Delta a\end{array}$ & $\begin{array}{c}\Delta \text { of (9) } \\
\Delta a=\frac{-\Delta R^{\uparrow}}{\sigma T_{S}^{4}-\sigma T_{a}^{4}}\end{array}$ & 0.021 \\
\hline $\begin{array}{c}\text { Longwave absorptance after the } \mathrm{CO} 2 \\
\text { doubling, } a^{\prime}\end{array}$ & $a^{\prime}=a+\Delta a$ & 0.821 \\
\hline $\begin{array}{c}\text { Change in } \varepsilon_{p} \text { due to a } \mathrm{CO}_{2} \text { doubling, } \\
\left(\Delta \varepsilon_{p}\right)_{e}\end{array}$ & (23) & -0.010 \\
\hline$\Delta T_{s} / T_{s}$ & (26) & 0.0045 \\
\hline$\Delta T_{a} / T_{a}$ & (27) & 0.0037 \\
\hline$\Delta T_{s}$ & $T_{S}\left(\Delta T_{S} / T_{S}\right)$ & $1.29 \mathrm{~K}$ \\
\hline$\Delta T_{a}$ & $T_{a}\left(\Delta T_{a} / T_{a}\right)$ & $0.91 \mathrm{~K}$ \\
\hline $\begin{array}{c}\text { Change in } \varepsilon_{p} \text { due to change in } \\
\text { temperatures, }\left(\Delta \varepsilon_{p}\right)_{i}\end{array}$ & (15) & -0.0014 \\
\hline $\begin{array}{l}\text { Feedback due to change in } \\
\text { temperatures, } f\end{array}$ & (8) & 0.122 \\
\hline
\end{tabular}
face gives

$$
\begin{gathered}
(1-a) \sigma T_{s}^{4}+a \sigma T_{a}^{4}=R^{\uparrow}=\sigma T_{e}^{4} \equiv \varepsilon_{p} \sigma T_{s}^{4} \\
\sigma T_{s}^{4}-a \sigma T_{a}^{4}=(1-b) \sigma T_{e}^{4}
\end{gathered}
$$

Table 2. Calculated Quantities.
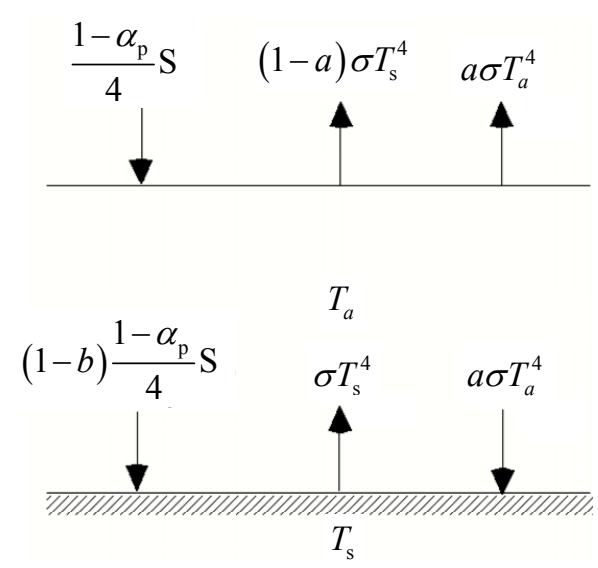

Figure 1. Schematic diagram of the solar and infrared fluxes in a one-layer atmosphere. 
where $R^{\uparrow}$ is the outgoing longwave radiation at TOA, and

$$
T_{e}=\left[\frac{\left(1-\alpha_{p}\right) S}{(4 \sigma)}\right]^{1 / 4}
$$

is the equivalent blackbody temperature of the planet.

Solving for $T_{s}$ and $T_{a}$ yields

$$
\begin{gathered}
T_{s}=\left(\frac{2-b}{2-a}\right)^{1 / 4} T_{e} \\
T_{a}=\left[\frac{a+b(1-a)}{a(2-a)}\right]^{1 / 4} T_{e}
\end{gathered}
$$

From Equation (9)

$$
\varepsilon_{p}=(1-a)+a\left(\frac{T_{a}}{T_{s}}\right)^{4}=\tau+a\left(\frac{T_{a}}{T_{s}}\right)^{4}
$$

where $\tau=1-a$ is the IR transmittance of the atmosphere. For fixed a,

$$
\left(\Delta \varepsilon_{p}\right)_{i}=4 a\left(\frac{T_{a}}{T_{s}}\right)^{4}\left[\frac{\Delta T_{a}}{T_{a}}-\frac{\Delta T_{s}}{T_{s}}\right]
$$

where subscript $i$ denotes "internal" in contrast to "external", for example, by changing the concentration of $\mathrm{CO}_{2}$. From Equation (8) with fixed $\alpha_{p}$, the necessary and sufficient condition for no TP feedback is $\Delta \varepsilon_{p}=0$, which by Equation (15) requires

$$
\frac{\Delta T_{a}}{T_{a}}=\frac{\Delta T_{s}}{T_{s}} \text { or, equivalently, } \Delta \log T_{a}=\Delta \log T_{s} .(16)
$$

This result is readily generalizable to an atmosphere with an arbitrary number of layers $\mathrm{K}$ by writing

$$
\varepsilon_{p}=\tau_{s}+\sum_{k=1}^{K} a_{k}\left(\frac{T_{k}}{T_{s}}\right)^{4}
$$

where $T_{k}$ is the temperature of layer $\mathrm{k}, a_{k}=\tau_{k-1 / 2}-\tau_{k+1 / 2}$, and $\tau_{j}$ is the transmittance from level $j$ to TOA. For fixed $\tau_{j}$,

$$
\left(\Delta \varepsilon_{p}\right)_{i}=4 \sum_{k=1}^{K} a_{k}\left(\frac{T_{k}}{T_{s}}\right)^{4}\left[\frac{\Delta T_{k}}{T_{k}}-\frac{\Delta T_{s}}{T_{s}}\right]
$$

Thus the sufficient condition for no TP feedback, $\left(\Delta \varepsilon_{p}\right)_{i}=0$, is

$$
\begin{aligned}
& \frac{\Delta T_{k}}{T_{k}}=\frac{\Delta T_{s}}{T_{s}} \text { for all } k \text { or, equivalently, } \\
& \Delta \log T_{k}=\Delta \log T_{s} \text { for all } k
\end{aligned}
$$

For all practical purposes this is also the necessary condition for no TP feedback.

From Equation (19), $\Delta T_{k} / \Delta T_{s}=T_{k} / T_{s}$ for all $k$. Thus, for no TP feedback the change in temperature with altitude, $\Delta T_{k}$, parallels the undisturbed temperature profile, $T_{k}$. Figure 2 shows $\Delta T_{k} / \Delta T_{s}=T_{k} / T_{s}$ for the US Standard Atmosphere. It is seen that for no TP feedback, $\Delta T_{k} / \Delta T_{s}=T_{k} / T_{s}$ decreases with increasing altitude in the troposphere and increases with altitude in the stratosphere. A similar decrease then increase is needed in the mesosphere and thermosphere, respectively. However, as can be seen from Equation (18), these regions are of less importance than the troposphere and stratosphere because of their smaller absorptance, $a_{k}$. Figure 2 shows that the temperature changes required for no TP feedback is less than the heretofore assumed uniform temperature change by as much as $25 \%$ at the tropopause and lower stratosphere.

Now suppose $\Delta T_{k}=\Delta T_{s}$ for all $\mathrm{k}$, as heretofore assumed for no feedback. Then Equation (18) yields

$$
\frac{\left(\Delta \varepsilon_{p}\right)_{i}}{\Delta T_{s}}=4 \sum_{k=1}^{K} a_{k}\left(\frac{T_{k}}{T_{s}}\right)^{4}\left[\frac{\mathrm{T}_{\mathrm{s}}-T_{k}}{T_{k} T_{s}}\right]
$$

Because $T_{s}>T_{k}$ for at least the part of the atmosphere where $a_{k}\left(T_{k} / T_{s}\right)^{4}$ is largest, namely, the troposphere and lower stratosphere, $\left(\Delta \varepsilon_{p}\right)_{i} / \Delta T_{s}>0$, hence by Equation (8) the feedback is negative, $f<0$, for a uniform temperature change and thus no change in lapse rate.

How must the lapse rate change for there to be no TP feedback? From the definition of lapse rate,

$$
\Gamma=\left(T_{k}-T_{k-1}\right) /\left(z_{k-1}-z_{k}\right)
$$

where $z$ is altitude and $\mathrm{k}$ increases from TOA to the surface, it is straightforward to show from Equation (19) that for no TP feedback,

$$
\frac{\Gamma^{\prime}}{\Gamma}=1+\frac{\Delta T_{s}}{T_{s}}
$$

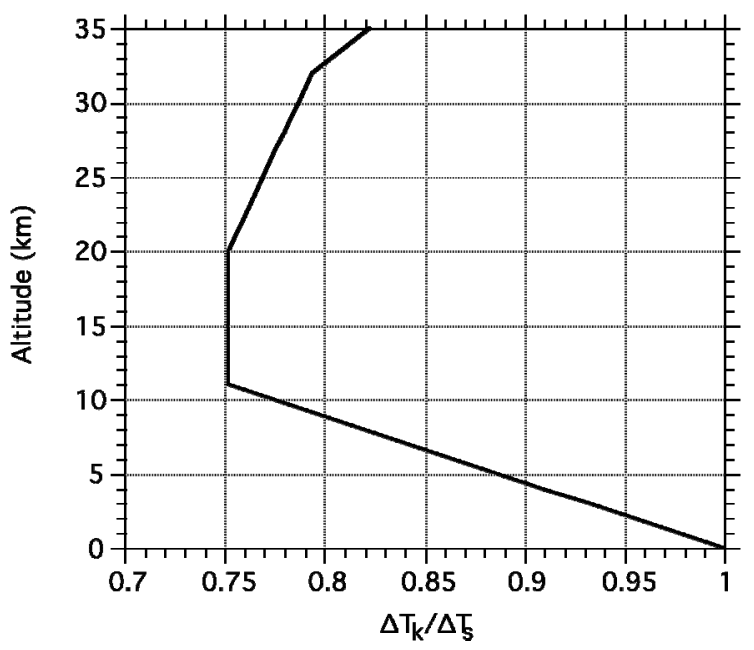

Figure 2. The vertical profile of $\Delta T(z) / \Delta T_{S}$ for zero TP feedback for the US Standard Atmosphere. 
where $\Gamma^{\prime}=\left[\left(T_{k}+\Delta T_{k}\right)-\left(T_{k-1}+\Delta T_{k-1}\right)\right] /\left(z_{k-1}-z_{k}\right)$.

Thus for no TP feedback the lapse rate must increase uniformly with altitude for surface warming, $\Delta T_{s}>0$, and must decrease uniformly with altitude for surface cooling, $\Delta T_{s}<0$. These changes in lapse rate are not large-for a $3^{\circ} \mathrm{C}$ global temperature change they are about $1 \%$ of the undisturbed lapse rate.

\section{Application to Solar Forcing}

We now return to the one-layer atmosphere of Figure 1. From Equations (11)-(13) for fixed $a$ and $b$ it is straightforward to show that

$$
\frac{\Delta T_{a}}{T_{a}}=\frac{\Delta T_{s}}{T_{s}}=\frac{\Delta T_{e}}{T_{e}}=\frac{1}{T_{e}} \Delta\left\{\left[\frac{\left(1-\alpha_{p}\right) S}{4 \sigma}\right]^{1 / 4}\right\}
$$

Thus the response of the atmospheric temperature to a change in $T_{a}$, either through a change in the solar irradiance or planetary albedo or both, satisfies the requirement for no TP feedback.

Figure 3 shows the profile of

$$
\left(\Delta T_{k} / T_{k}\right) /\left(\Delta T_{s} / T_{s}\right)
$$

in response to a $2 \%$ increase in the solar irradiance calculated by our 26-layer stratosphere/troposphere radiative-convective model [3] with the convective adjustment turned off and no temperature dependence of the infrared transmittances. It is seen that

$$
\left(\Delta T_{k} / T_{k}\right) /\left(\Delta T_{s} / T_{s}\right)=1
$$

for all 26 layers, hence TP feedback is zero for solar forcing.

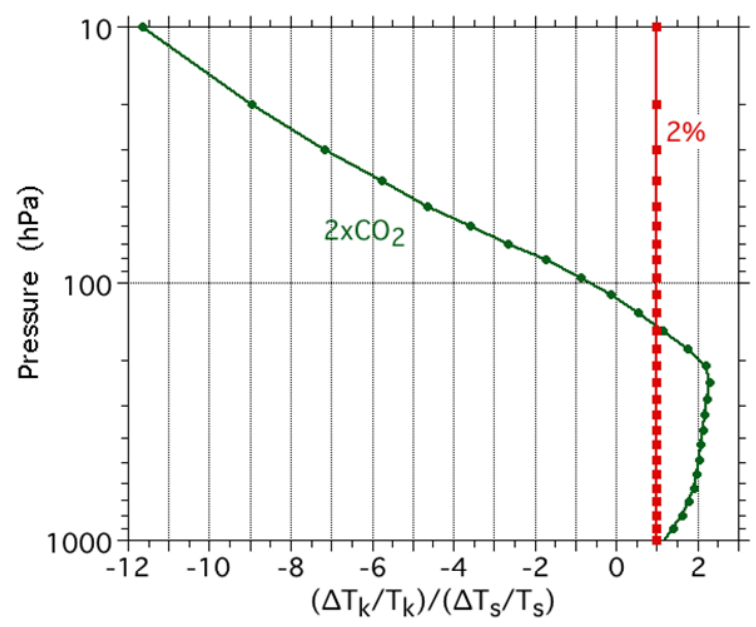

Figure 3. Vertical profile of $\left(\Delta T_{k} / T_{k}\right) /\left(\Delta T_{s} / T_{s}\right)$ versus pressure simulated for a $2 \%$ increase in solar irradiance and a $\mathrm{CO}_{2}$ doubling by a 26-layer stratosphere/troposphere radiative-convective model [3] with the convective adjustment turned off and no temperature dependence of the longwave transmittances.

\section{Application to Infrared Forcing}

In this section we show that condition (16) for no TP feedback is not satisfied for radiative forcing in the infrared, such as from changing the concentration of greenhouse gases, which changes $a$ to $a^{\prime}=a+\Delta a$. By Equation (14) this will change the planetary emissivity by

$$
\left(\Delta \varepsilon_{p}\right)_{e}=-\left[1-\left(\frac{T_{a}}{T_{s}}\right)^{4}\right] \Delta a
$$

where the subscript e denotes an "external" change.

The new equilibrium is given by Equations (9) and (10) with $a$ replaced by $a^{\prime}, T_{s}$ by $T_{s}+\Delta T_{s}$, and $T_{a}$ by $T_{a}+\Delta T_{a}$. This yields after using the binomial expansion and linearizing, $(1+\Delta x / x)^{4} \cong 1+4 \Delta x / x$,

$$
\begin{gathered}
{\left[1-a^{\prime}\right] T_{s}^{3} \Delta T_{s}+a^{\prime} T_{a}^{3} \Delta T_{a}=\frac{\Delta a}{4}\left(T_{s}^{4}-T_{a}^{4}\right)} \\
T_{s}^{3} \Delta T_{s}-a^{\prime} T_{a}^{3} \Delta T_{a}=\frac{\Delta a}{4} T_{a}^{4}
\end{gathered}
$$

Solving these Equations yields

$$
\begin{gathered}
\frac{\Delta T_{s}}{T_{s}}=\frac{\Delta a}{4\left(2-a^{\prime}\right)} \\
\frac{\Delta T_{a}}{T_{a}}=\frac{\Delta a}{4 a^{\prime}}\left[\frac{\left(T_{s} / T_{a}\right)^{4}}{2-a^{\prime}}-1\right]
\end{gathered}
$$

From Equations (26) and (27), together with Equations (12) and (13), we obtain

$$
\frac{\Delta T_{a} / T_{a}}{\Delta T_{s} / T_{s}}=\frac{1}{a^{\prime}}\left[\frac{a(2-b)}{a+b(1-a)}-\left(2-a^{\prime}\right)\right]
$$

Setting Equation (28) equal to unity, assuming $a^{\prime} \cong a$ and solving yields two solutions, $b=0$ and $a=2$, the former having an unphysical solar absorptance and the latter an unphysical infrared absorptance. Consequently, for the case of infrared forcing, the condition required for zero TP feedback given by Equation (16) cannot be satisfied.

The values of $\Delta T_{a} / T_{a}, \Delta T_{s} / T_{s},\left(\Delta \varepsilon_{p}\right)_{i}$ and $f$ calculated for a $\mathrm{CO}_{2}$ doubling for the prescribed values shown in Table 1 are presented in Table 2. It can be seen that the change in the temperature profile yields a negative $\left(\Delta \varepsilon_{p}\right)_{i}$ which is the same sign as the change in $\left(\Delta \varepsilon_{p}\right)_{e}$ due to the doubling of the $\mathrm{CO}_{2}$ concentration. As a result the feedback is positive and rather large.

This positive TP feedback increases the surface temperature change by $16 \%$ from its zero feedback value of $1.11 \mathrm{~K}$ to $1.29 \mathrm{~K}$. It occurs even in the absence of a stratosphere, which the one-layer atmospheric model does not possess. The presence of a stratosphere would 
all the more result in TP feedback because the sign of the stratospheric temperature changes induced by a change in greenhouse-gas concentration is opposite to the sign of the tropospheric temperature changes, thereby not satisfying condition (19) for no TP feedback.

Figure 3 shows the profile of $\left(\Delta T_{k} / T_{k}\right) /\left(\Delta T_{s} / T_{s}\right)$ in response to a doubling of the $\mathrm{CO}_{2}$ concentration calculated by our 26-layer stratosphere/troposphere radiativeconvective model [3] with the convective adjustment turned off and no temperature dependence of the longwave transmittances. It is seen that

$$
\left(\Delta T_{k} / T_{k}\right) /\left(\Delta T_{s} / T_{s}\right) \neq 1
$$

for all layers, hence the TP feedback is not zero. As shown in Table 2, $f=0.122$

\section{Conclusions}

This study has shown the following: 1) the heretofore assumed condition for no temperature-profile (TP)/lapserate feedback, $\Delta T(z)=\Delta T_{s}$ for all altitudes $z$, which gives no change in lapse rate, $\Delta(\mathrm{d} T(z) / \mathrm{d} z)=0$, in fact yields a negative feedback; 2 ) the correct condition for no TP feedback is $\Delta T(z) / T(z)=\Delta T_{s} / T_{s}$ for all $z ; 3$ ) this condition translates into a uniform increase (decrease) in lapse rate with altitude for an increase (decrease) in surface temperature; 4) the temperature changes caused by a change in solar irradiance and/or planetary albedo satisfy the condition for no TP feedback; and 5) the temperature changes caused by a change in greenhouse gas concentration do not satisfy the condition for no TP feedback and, instead, yield a positive feedback.

\section{Acknowledgements}

This material is based upon work supported by the National Science Foundation under Award No. ATM0084270. Any opinions, findings, and conclusions or recommendations expressed in this publication are those of the authors and do not necessarily reflect the views of the National Science Foundation.

\section{REFERENCES}

[1] R. D. Cess, "Global Climate Change: An Investigation of Atmospheric Feedback Mechanisms," Tellus, Vol. 27, No. 3, 1975, pp. 193-198. doi:10.1111/j.2153-3490.1975.tb01672.x

[2] A. Arking, "The Radiative Effects of Clouds and Their Impact on Climate," Bulletin of the American Meteorological Society, Vol. 72, No. 6, 1991, pp. 795-813. doi:10.1175/1520-0477(1991)072<0795:TREOCA $>2.0 . \mathrm{C}$ $\underline{\mathrm{O} ; 2}$

[3] C. B. Entwistle, "Analysis of the Nature of Zero Feedback in the Climate System Using a Multilayer Radiative-Convective Model," M.S. Thesis, University of Illinois at Urbana-Champaign, Urbana and Champaign, 1992, p. 201.

[4] M. E. Schlesinger, "Feedback Analysis of Results from Energy Balance and Radiative-Convective Models," In: M. C. MacCracken and F. M. Luther, Eds., The Potential Climatic Effects of Increasing Carbon Dioxide, U. S. Department of Energy, 1985, pp. 280-319.

[5] M. E. Schlesinger, "Quantitative Analysis of Feedbacks in Climate Model Simulations of $\mathrm{CO}_{2}$-Induced Warming," In: M. E. Schlesinger, Ed., Greenhouse-Gas-Induced Climatic Change: A Critical Appraisal of Simulations and Observations, Elsevier, Amsterdam, 1988, pp. 653-737.

[6] M. E. Schlesinger, "Quantitative Analysis of Feedbacks in Climate Model Simulations," In A. Berger, R. E. Dickinson and J. W. Kidson, Eds., Understanding Climate Change, American Geophysical Union, Washington DC 1989, pp. 177-187. doi:10.1029/GM052p0177

[7] G. Myhre, E. J. Highwood, K. P. Shine and F. Stordal, "New Estimates of Radiative Forcing Due to Well Mixed Greenhouse Gases," Geophysical Research Letters, Vol. 25, No. 14, 1998, pp. 2715-2718. doi:10.1029/98GL01908 